\title{
Glutathione and the redox control system trypanothione/trypanothione reductase are involved in the protection of Leishmania spp. against nitrosothiol-induced cytotoxicity
}

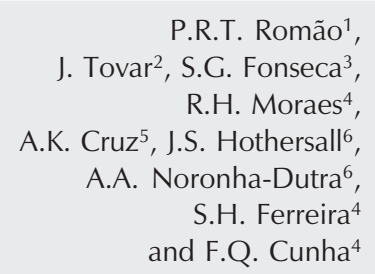

P.R.T. Romão ${ }^{1}$,

J. Tovar' ${ }^{2}$, S.G. Fonseca ${ }^{3}$,

R.H. Moraes ${ }^{4}$,

A.K. Cruz ${ }^{5}$, J.S. Hothersall6,

A.A. Noronha-Dutra ${ }^{6}$,

S.H. Ferreira ${ }^{4}$

and F.Q. Cunha ${ }^{4}$

\author{
${ }^{1}$ Laboratório de Imunoparasitologia, Curso de Medicina, \\ Universidade do Sul de Santa Catarina (UNISUL), Tubarão, SC, Brasil \\ ${ }^{2}$ School of Biological Sciences, Royal Holloway University of London, Egham, UK \\ ${ }^{3}$ Laboratório de Imunologia, Instituto do Coração, Faculdade de Medicina, \\ Universidade de São Paulo, São Paulo, SP, Brasil \\ ${ }^{4}$ Departamento de Farmacologia, ${ }^{5}$ Departamento de Biologia Celular e Molecular e \\ Bioagentes Patogênicos, Faculdade de Medicina de Ribeirão Preto, de São Paulo, \\ Ribeirão Preto, SP, Brasil \\ ${ }^{6}$ The Institute of Urology and Nephrology, University College of London, London, UK
}

\section{Correspondence}

P.R.T. Romão

Laboratório de Imunoparasitologia

Curso de Medicina, UNISUL

Rua José Acácio Moreira, 787,

DEHON

88704-900 Tubarão, SC

Brasil

Fax: +55-48-621-3067

E-mail: ptorres@unisul.br

Research supported by FAPESP (No. 97/01938-8). P.R.T. Romão and

S.G. Fonseca were recipients of FAPESP fellowships. F.Q. Cunha, S.H. Ferreira, and R.H. Moraes were recipients of CNPq fellowships.

Received January 21, 2005 Accepted November 11, 2005

\begin{abstract}
Glutathione is the major intracellular antioxidant thiol protecting mammalian cells against oxidative stress induced by oxygen- and nitrogen-derived reactive species. In trypanosomes and leishmanias, trypanothione plays a central role in parasite protection against mammalian host defence systems by recycling trypanothione disulphide by the enzyme trypanothione reductase. Although Kinetoplastida parasites lack glutathione reductase, they maintain significant levels of glutathione. The aim of this study was to use Leishmania donovani trypanothione reductase gene mutant clones and different Leishmania species to examine the role of these two individual thiol systems in the protection mechanism against S-nitroso- $N$-acetyl-D,L-penicillamine (SNAP), a nitrogen-derived reactive species donor. We found that the resistance to SNAP of different species of Leishmania was inversely correlated with their glutathione concentration but not with their total low-molecular weight thiol content (about $0.18 \mathrm{nmol} / 10^{7}$ parasites, regardless Leishmania species). The glutathione concentration in $L$. amazonensis, L. donovani, L. major, and L. braziliensis were 0.12 , $0.10,0.08$, and $0.04 \mathrm{nmol} / 10^{7}$ parasites, respectively. L. amazonensis, that have a higher level of glutathione, were less susceptible to SNAP (30 and $100 \mu \mathrm{M}$ ). The $\mathrm{IC}_{50}$ values of SNAP determined to L. amazonensis, L. donovani, L. major, and L. braziliensis were 207.8, 188.5, 160.9 , and $83 \mu \mathrm{M}$, respectively. We also observed that $L$. donovani mutants carrying only one trypanothione reductase allele had a decreased capacity to survive ( $40 \%)$ in the presence of SNAP (30-150 $\mu \mathrm{M})$. In conclusion, the present data suggest that both antioxidant systems, glutathione and trypanothione/trypanothione reductase, participate in protection of Leishmania against the toxic effect of nitrogen-derived reactive species.
\end{abstract}

Key words

- Leishmania

- Glutathione

- Trypanothione

- Trypanothione reductase

- Nitric oxide

- Free radicals 


\section{Introduction}

The trypanosomatids, members of the order Kinetoplastida, include parasitic protozoa of importance to public health such as Leishmania spp. Leishmania cause a spectrum of diseases ranging from self-healing ulcers to disseminated and often fatal infections, depending on the species involved and the host's immune response. Adequate vaccines against trypanosomatid infections have yet to be developed, and drugs currently available for chemotherapeutic intervention are mostly unsatisfactory mainly because of their lack of specificity, toxicity to humans, and, in many cases, developed parasite resistance (1). Thus, one of the priorities in tropical medicine research has been the identification and characterisation of parasite-specific biomolecules, which play relevant physiological roles and thus might be exploited as selective targets.

Among many other metabolic distinctions, trypanosomatids maintain their intracellular redox balance by a mechanism that is different from that of their insect vectors and mammalian hosts. They lack glutathione reductase, which in nearly all other organisms is responsible for the maintenance of an intracellular thiol-reducing environment, and thus for the reduction of disulphides, detoxification of peroxides and synthesis of DNA precursors (2). Instead, they possess a unique system using trypanothione $\left[\mathrm{T}(\mathrm{SH})_{2}\right]$ that is the major reduced thiol of Kinetoplastida parasites (3) and comprises a spermidine moiety linked to two glutathione molecules (2). Together with three thiol-redox proteins, trypanothione reductase (TryR), tryparedoxin and tryparedoxin peroxidase $(4,5), \mathrm{T}(\mathrm{SH})_{2}$ is thought to provide defence against oxidants, certain heavy metals (6) and xenobiotics (3). Thus, TryR has a vital physiological role in maintaining $\mathrm{T}(\mathrm{SH})_{2}$ redox, particularly within the highly oxidative intracellular environment of the host cells which is generated during the antimicrobial defence response.

In murine leishmaniasis, nitric oxide (NO) plays a crucial role in the killing of parasites both in vitro $(7)$ and in vivo $(8,9)$. In vitro macrophage microbicidal activity correlates with NO production, and both in vivo and in vitro microbicidal activities are completely inhibited by the NO synthase inhibitor Larginine analogue $\mathrm{N}^{\mathrm{G}}$-monomethyl-L-arginine (L-NMMA) but not by its enantiomer D-NMMA (8). In addition, the NO donors, S-nitroso- $N$-acetyl-D,L-penicillamine (SNAP) and 3-morpholino-sydnonimine hydrochloride are able to kill Leishmania parasites in a cell-free model system (10).

We have reported that glutathione is involved in the protection of mammalian macrophages against the cytotoxic effects of NO. Furthermore, despite evidence that glutathione in Leishmania appears not to be the major antioxidant, we have demonstrated that it protects $L$. major from the toxic effects of NO (11).

In the present study, we have extended these observations by comparing the glutathione levels and SNAP sensitivity of different Leishmania species: L. amazonensis, L. braziliensis, L. donovani, and L. major. Our results demonstrate that the sensitivity of distinct species of Leishmania to SNAP is inversely correlated with their glutathione concentration. When we extended our investigation to the role of TryR in the protection against SNAP using mutants of $L$. donovani for the TryR gene (tryR, formerly tryA) (12), we found that, compared to control parasites (try $R$ genotype $\mathrm{e}^{++^{+}+}$), a double mutant clone (try $R$ genotype ${ }^{-r^{+}}$) was significantly more sensitive to the cytotoxic effect of SNAP. Overall, these results demonstrate that glutathione as well as the $\mathrm{T}(\mathrm{SH})_{2} /$ TryR redox system are essential protective components against NO cytotoxicity in Leishmania.

\section{Material and Methods}

\section{Leishmania strains and culture conditions}

The Leishmania species used in this study 
were L. braziliensis (MHOM/BR/75/M2904), L. amazonensis (MPRO/BR/72/M1841-LV79), L. major (LV-39, clone 5-Rho-SU/59/P), and $L$. donovani (clone LV9-3 from MHOM/ ET/67/HU3). The $L$. donovani clones used were: wild-type LV9-3, which possesses three copies of the tryR (formerly tryA) gene $\left(\right.$ try $\left.R^{+/+/+}\right)$and the mutants of TryR: clones H2-try $R^{-1+++}$ (LV9-3 submitted to single replacement), clone HB3-try $R^{-1-l_{+}}$(LV9-3 submitted to double replacement (12) and clone HB3-pTTcTR (Tovar J and Fairlamb AH, unpublished results) that is identical to clone HB3 $\left(\right.$ try $R^{-1 /+}$ ) but harbours plasmid pTTcTR (13). Promastigote forms of all Leishmania species were grown in M199 medium supplemented with $40 \mathrm{mM}$ HEPES, $\mathrm{pH}$ 7.4, $0.1 \mathrm{mM}$ adenine, $7.7 \mathrm{mM}$ hemin, $10 \%$ (v/v) heat-inactivated foetal calf serum, $50 \mathrm{U} / \mathrm{mL}$ penicillin, and $50 \mu \mathrm{g} / \mathrm{mL}$ streptomycin. Cultures were incubated at $26^{\circ} \mathrm{C}$, and cells were kept at densities ranging between $5 \times 10^{5}$ and $3 \times 10^{7}$ parasites $/ \mathrm{mL}$. Transfectants were cultured in the presence of selective drugs (12). The mutant $\mathrm{H} 2-$ try $R^{-/+++}$was cultured in the presence of $16 \mu \mathrm{g} / \mathrm{mL}$ hygromycin $\mathrm{B}$, the clone HB3try $R^{-I_{+}+}$in the presence of $16 \mu \mathrm{g} / \mathrm{mL}$ hygromycin B plus $2.5 \mu \mathrm{g} / \mathrm{mL}$ phleomycin, and the HB3-try $R^{--/+}$clone plus episomal pTTcTR in the presence of $16 \mu \mathrm{g} / \mathrm{mL}$ hygromycin B, $25 \mu \mathrm{g} / \mathrm{mL}$ G418 and $2.5 \mu \mathrm{g} /$ $\mathrm{mL}$ phleomycin.

\section{Growth curves}

Promastigotes of wild-type or L. donovani mutants were cultured in M199 medium prepared as described previously (14). Cell density in the inoculum was $1 \times 10^{5} / \mathrm{mL}$. Viability was evaluated from motility and cell density was determined daily using a hemocytometer.

\section{Glutathione and non-protein low-molecular weight thiol measurement}

Non-protein low-molecular weight thi- ols and glutathione (reduced plus disulphide forms) were measured in lysates of promastigote forms (stationary phase) of different Leishmania species (L. amazonensis, L. braziliensis, $L$. donovani, and L. major) including the different clones of $L$. donovani. Lowmolecular weight thiols were measured using Ellman's reagent (15). To measure soluble thiols the samples were deproteinised with $1 \%$ sulfosalicylic acid in the presence of $5 \mathrm{mM}$ EDTA. The concentration of $\mathrm{SH}$ groups was calculated from a standard curve of 0.01 to $2 \mathrm{nmol}$ cysteine. Glutathione levels were measured by the glutathione reductase enzyme recycling method (16). These assays were adapted for use in a microtitre plate using a microplate spectrophotometer system spectra MAX 250 (Molecular Devices, Union City, CA, USA). Cells were lysed by the addition of $100 \mu \mathrm{L} 1 \mathrm{mM}$ EDTA to each well and freezing immediately. Following thawing, plates were shaken for $30 \mathrm{~s}$ and then sonicated for $60 \mathrm{~s}$. Assays were carried out immediately (17). Although this assay provides a measure of both oxidised and reduced glutathione, in non-oxidative stress equilibrium, the cellular condition under which we have measured glutathione, the thiol content is $95-99 \%$ reduced glutathione. Cytotoxic effect of S-nitroso- $N$-acetyl-D,L-
penicillamine on Leishmania viability

The direct cytotoxic effect of the nitrosothiol SNAP on Leishmania species was measured. Briefly, parasites (3 x 10\%/well) were incubated in M199 medium supplemented with $10 \%$ heat-inactivated foetal calf serum in the presence or absence of SNAP (30$1000 \mu \mathrm{M}$ ) for $12 \mathrm{~h}$. The incubation medium contains L-cystine which allows the membrane transport of SNAP into cells (18). Parasites were then pulsed with $1 \mu \mathrm{Ci} /$ well $\left[{ }^{3} \mathrm{H}\right]$-thymidine, and the incorporation of radioactivity by viable parasites was determined after $24 \mathrm{~h}$ in a $\beta$-counter (11). The 
$50 \%$ inhibitory concentration $\left(\mathrm{IC}_{50}\right)$ values for each Leishmania species were determined using Sigma-Plot software, Version 5.0.

\section{Statistical analysis}

Data are reported as means \pm SEM and statistical significance $(\mathrm{P}<0.05)$ was assessed by ANOVA followed by Bonferroni's $t$-test.

\section{Results}

Non-protein low-molecular weight thiol and glutathione concentration in different Leishmania species

Glutathione concentrations of different species of Leishmania (L. amazonensis, $L$.

Figure 1. Intracellular glutathione (GSH) concentrations in different Leishmania species. Total glutathione (GSH + oxidized glutathione (GSSG)) was measured in lysates of $1 \times 10^{7}$ promastigotes of different Leishmania species ( $L$. amazonensis, L. braziliensis, L. donovani, and L. major). Data are reported as means \pm SEM of four replicate cultures and are representative of three experiments. ${ }^{*} \mathrm{P}<0.05 \mathrm{com}-$ pared with $L$. amazonensis; ${ }^{\#} \mathrm{P}<0.05$ compared with $L$. donovani; ${ }^{+} \mathrm{P}<0.05$ compared with L. major (ANOVA followed by Bonferroni's $t$-test).

Figure 2. Concentrations of nonprotein low-molecular weight thiols in different Leishmania species. Total non-protein thiols (glutathione, $\mathrm{T}(\mathrm{SH})_{2}$, cysteine, and ovothiol) were measured in lysates of $1 \times 10^{7}$ promastigotes of Leishmania species (L. amazonensis, L. braziliensis, L. donovani, and $L$. major). Data are reported as means \pm SEM of four replicate cultures and are representative of three experiments.
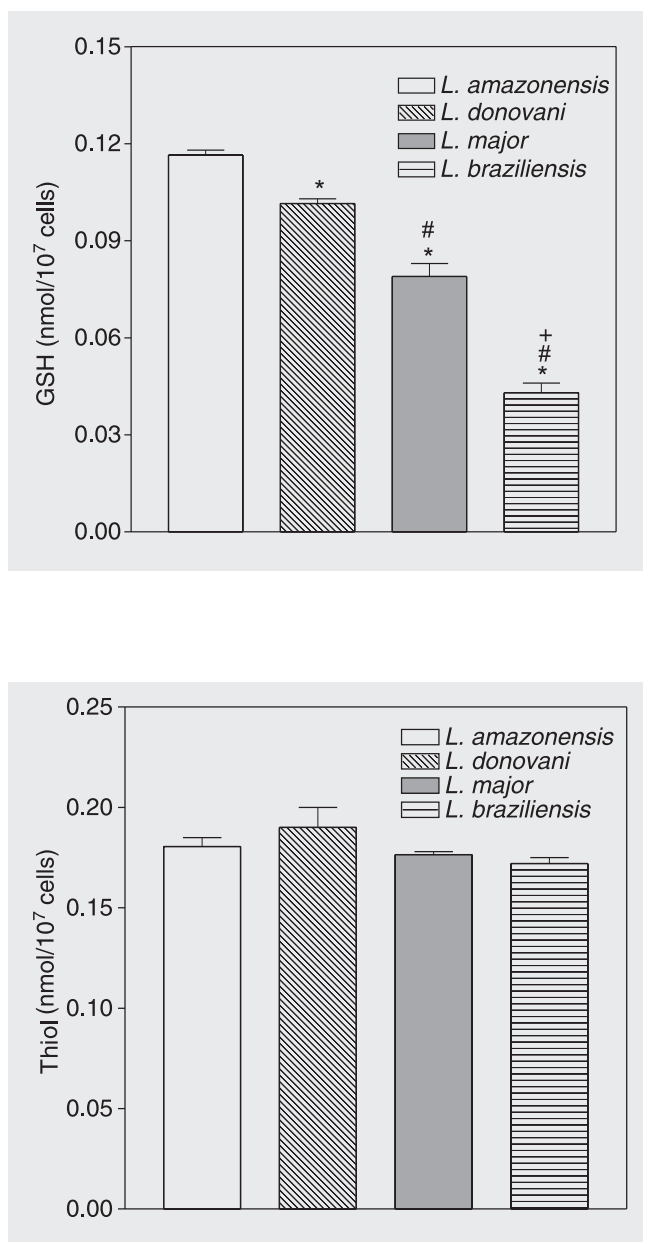

braziliensis, L. major, and L. donovani) are shown in Figure 1. Statistically significant differences in glutathione levels were observed between all Leishmania species, with promastigotes of L. braziliensis having the lowest level. Glutathione concentration was L. amazonensis $>$ L. donovani $>$ L. major $>$ L. braziliensis. No significant differences in the non-protein low-molecular weight thiol levels were detected between different Leishmania species (Figure 2).

Cytotoxic effect of S-nitroso- $\mathrm{N}$-acetyl-D, Lpenicillamine on different Leishmania species

To ascertain whether the glutathione levels in the different Leishmania species (Figure 1) correlate with their sensitivity to reactive nitrogen species, we investigated the cellular viability of $L$. amazonensis, $L$. braziliensis, L. donovani, and L. major after treatment with SNAP. The addition of a SNAP directly to promastigotes of different species of Leishmania resulted in dose-dependent parasite killing (Figure 3). The sensitivity of Leishmania species to SNAP correlated inversely with the glutathione levels. L. amazonensis, that has a higher glutathione concentration, was more resistant to the cytotoxic effect of SNAP at concentrations of 30 and $100 \mu \mathrm{M}$, with an $\mathrm{IC}_{50}$ of 207.8 (200.2-215.7 $\mu \mathrm{M})$. In contrast, L. braziliensis, that shows lower levels of glutathione, was more susceptible to the toxic effect of SNAP $\left(\mathrm{IC}_{50}\right.$ of $\left.83(79.7-85.5 \mu \mathrm{M})\right)$. The $\mathrm{IC}_{50}$ values determined for $L$. donovani and L. major were 188.5 and $160.9 \mu \mathrm{M}$, respectively.

Effect of S-nitroso- $\mathrm{N}$-acetyl-D, L-penicillamine on the viability of Leishmania donovani tryR gene mutants

To ascertain whether the $\mathrm{T}(\mathrm{SH})_{2} / \operatorname{TryR}$ antioxidant system is involved in the protection of Leishmania against nitrogen-derived reactive species, we investigated the effect 
of SNAP on the viability of targeted L. donovani TryR mutants that have been generated by gene disruption. Wild-type $L$. donovani (LV9-3, genotype of tryR $R^{+/++}$) and clones submitted to a single (clone $\mathrm{H} 2$ genotype of try $R^{-/++}$) or double (clone HB3 genotype of $\operatorname{try} R^{-I-+}$ ) experiment for the replacement of the $\operatorname{try} R$ locus were utilised. It is important to mention that a null mutant has not yet been obtained for $\operatorname{try} R(12,19)$. The addition of SNAP directly to promastigotes of different L. donovani clones resulted in partial parasite killing (Figure 4). Recombinant HB3 possessing only one tryR allele was more sensitive to the toxic effect of SNAP at concentrations of 30-150 $\mu \mathrm{M}$ compared with recombinant $\mathrm{H} 2$ or $L$. donovani parental clone (LV9-3). The sensitivity to high concentration of SNAP $(300 \mu \mathrm{M})$ was unchanged. To demonstrate that the decrease in the sensitivity was solely due to the disruption of the tryR gene, we tested the viability of clone HB3 $\left(\right.$ try $\left.R^{-/ /+}\right)$supplemented with the plasmid PTTcTR that harbours a functional $T$. cruzi tryR gene (13). This recombinant strain, whose levels of TryR are higher than those of wild-type parasites, regained wild-type levels of resistance to SNAP-generated stress (Figure 4).

\section{Glutathione concentration in Leishmania donovani TryR mutants}

Our previous results (11) and the data presented here suggest that glutathione is involved in the protection of Leishmania against the toxic effects of SNAP. As TryR is the enzyme that maintains $\mathrm{T}(\mathrm{SH})_{2}$ in its reduced form and plays a central role in oxidant detoxification through the enzymatic regeneration of the thiol pool, we examined whether the effect of the loss of the tryR copy in L. donovani mutant clones changed the glutathione levels. No significant differences in glutathione were detected between the wild-type and single- or double-targeted $L$. donovani tryR mutants or with the HB3-

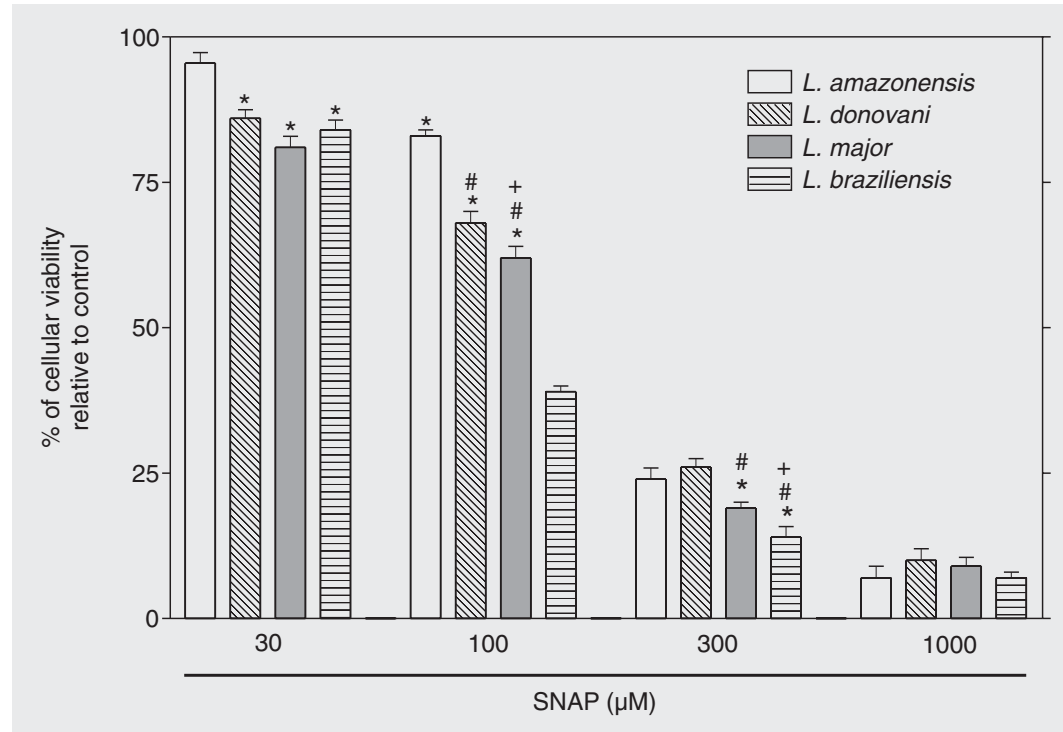

Figure 3. Cytotoxic effect of S-nitroso- $N$-acetyl-D,L-penicillamine (SNAP) on different Leishmania species. Promastigote forms of $L$. amazonensis, $L$. braziliensis, $L$. donovani, and $L$. major ( $3 \times 10^{6}$ cells/well) were incubated in M199 medium (control) or M199 plus SNAP (30$1000 \mu \mathrm{M})$. The cultures were pulsed with ${ }^{3}[\mathrm{H}]$-thymidine $12 \mathrm{~h}$ after SNAP treatment. Leishmania survival was determined after $24 \mathrm{~h}$ of further culture by the ability of residual live parasites to incorporate ${ }^{3}[\mathrm{H}]$-thymidine. Data are reported are means \pm SEM of four replicates and are representative of three experiments. Statistically significant different with ${ }^{*} \mathrm{P}<0.05$ compared with $L$. amazonensis, ${ }^{\mathrm{P}}<0.05$ compared with $L$. donovani, and ${ }^{+} \mathrm{P}<$ 0.05 compared with $L$. major (ANOVA followed by Bonferroni's $t$-test).

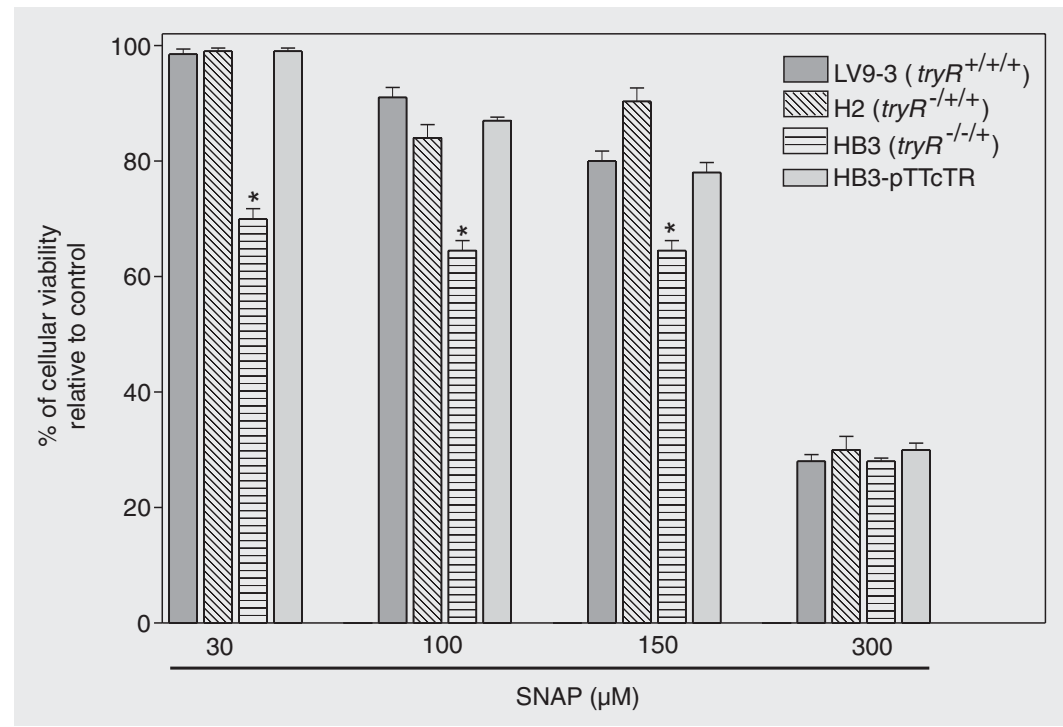

Figure 4. Cytotoxic effect of S-nitroso- $N$-acetyl-D,L-penicillamine (SNAP) on L. donovani viability. Effects of the number of tryR allele promastigotes $\left(3 \times 10^{6}\right)$ of wild-type $L$. donovani (LV9-3: try $R^{+/+/+}$) and clones H2 (try $R^{-/+/+}$), HB3 (try $R^{-/ /+}$) and HB3-pTTcTR (try $R^{-/ /+}$plus PTEXTcTR episomic) were incubated in M199 medium (control) or M199 plus SNAP (30$300 \mu \mathrm{M})$ for $12 \mathrm{~h}$. Leishmania survival was determined after $24 \mathrm{~h}$ of further culture by the ability of residual live parasites to incorporate ${ }^{3}[\mathrm{H}]$-thymidine. Data are reported as means \pm SEM of four replicates and are representative of three experiments. ${ }^{*} \mathrm{P}<0.05$ compared with the parental clone (LV9-3; ANOVA followed by Bonferroni's $t$-test). 
pTTcTR transfected clone (Figure 5).

\section{Growth of Leishmania donovani clones}

Growth curves of both wild-type (LV9-3) and $L$. donovani mutants (clones $\mathrm{H} 2-$ try $R^{-/+/+}$,

Figure 5. Intracellular glutathione (GSH) levels in L. donovani trypanothione reductase (try $R$ ) mutants. Total glutathione (GSH + oxidized glutathione (GSSG)) was measured in lysates of $1 \mathrm{x}$ $10^{7}$ promastigotes of wild-type L. donovani (LV9-3: try $R^{+/+/+}$) and clones $\mathrm{H} 2\left(\right.$ try $\left.^{-/+/+}\right)$, HB3 $\left(\right.$ try $\left.R^{-/-1+}\right)$, and HB3-pTTcTR (try $R^{-/ /+}$plus pTEXTcTR episomic). Data are reported as means \pm SEM of four replicate cultures and are representative of three experiments.
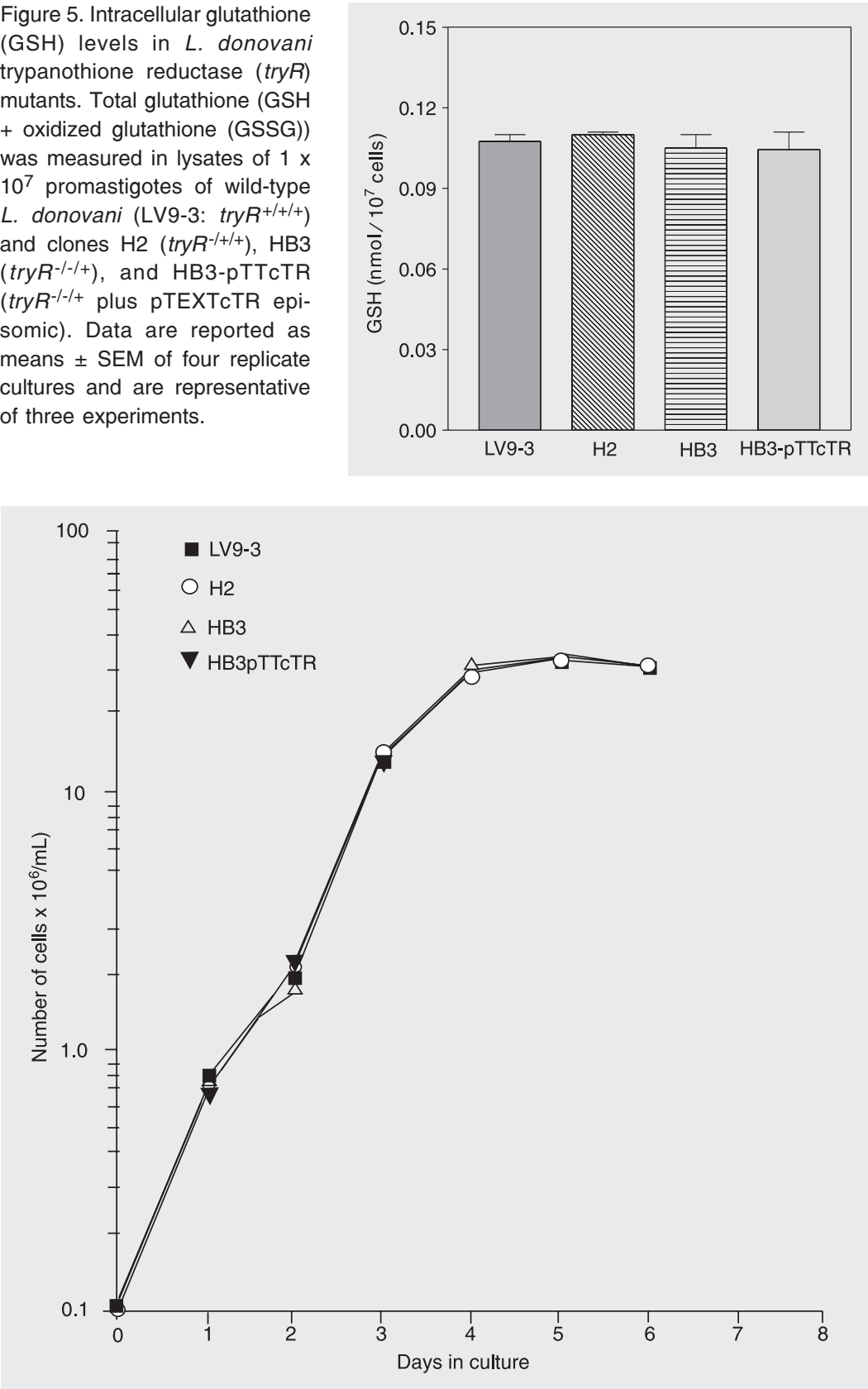

Figure 6. Growth curve of wild-type L. donovani (LV9-3: try $R^{+/+/+}$) and clones $\mathrm{H} 2$ (try $R^{-/+/+}$), HB3 $\left(\right.$ try $R^{-/ /+}$), and HB3-pTTcTR (try $R^{-/ /+}$plus pTEXTcTR episomic). Each point represents the average of counts from two cultures, initially inoculated with $1 \times 10^{5}$ organisms $/ \mathrm{mL}$ in M199 medium.
HB3-tryR $R^{-I-+}$ and HB3-pTTcTR) show the typical log and stationary growth phases. A similar pattern of continual growth was observed for all transfectants (Figure 6).

\section{Discussion}

The results presented in this study suggest that both glutathione and $\mathrm{T}(\mathrm{SH})_{2} /$ TryR antioxidant systems are important components of the protective mechanisms of Leishmania against the cytotoxic effects of nitrogen-derived reactive species. This conclusion is supported by the following observations: a) the sensitivity of distinct species of Leishmania (L. amazonensis, L. braziliensis, L. donovani, and L. major) to SNAP, a nitrogen-derived reactive species donor, correlated inversely with their cellular glutathione levels but not with their total non-protein low-molecular weight thiol content; b) mutants of $L$. donovani possessing only one wild-type tryR allele, $\operatorname{try} R^{-/ /+}$(submitted to a double replacement of the tryR gene locus), had a decreased capacity to survive in the presence of SNAP. Nevertheless, under normal conditions of in vitro growth, the $L$. donovani wild-type and recombinant mutants have similar levels of glutathione.

Our data are consistent with Lemesre et al. (20) who demonstrated that amastigote forms of L. amazonensis, which have higher levels of glutathione, are particularly resistant to NO killing. It is recognised that similar molecular targets of $\mathrm{NO}$ are present in microorganisms and in mammalian cells $(21,22)$ and, it has been demonstrated that glutathione protects several mammalian cells, including macrophages, against the oxidative stress induced by oxygen (23) and nitrogen-derived oxidants $(11,24,25)$. Thus, the different intracellular glutathione levels may explain the large differences in susceptibility to SNAP among different species of Leishmania. The observation that the levels of non-protein thiols were similar in all Leishmania species analysed supports this con- 
clusion. Furthermore, it has been demonstrated that buthionine sulfoximine, an inhibitor of glutathione synthesis, protects mice from Trypanosoma brucei infections by lowering the parasites' glutathione and protein thiol levels (26). In addition, conjugation of electrophilic drugs with glutathione via glutathione S-transferase has been shown to be an important detoxification system in T. cru$z i$ epimastigotes (27).

The mechanism by which glutathione is implicated in the protection of trypanosomatid protozoa against the cytotoxic effects of nitrogen-derived oxidants is not clear. However, there is evidence that biological NOderived oxidants such as nitrogen dioxide and carbonate radical anion dioxide promote cell injury (28-30). These events depend on the intracellular concentration of low-molecular weight thiols such as glutathione $(24,31,32)$. Furthermore, glutathione and protein-cysteines are the preferred targets of NO-derived oxidants being oxidised to the respective thyil radicals $(24,33)$.

$\mathrm{T}(\mathrm{SH})_{2}$, a glutathione and spermidine conjugate, is one of the unique features of Kinetoplastida. In trypanosomatids, the existence of TryR instead of glutathione reductase suggested that the thiol pool in these organisms is dependent on TryR (3). Thus, TryR has long been regarded as a target molecule for selective attack of the trypanosomatids. Our data demonstrate that the $L$. donovani recombinant HB3 clone possessing only one wild-type try $R$ allele, retaining only one-third of its original TryR activity (12), has a partially decreased capacity to survive in the presence of SNAP. Furthermore, this mutant and the L. donovani mutants TR/TR::hyg and TR/TR::hyg/TR::neo, which also carry a single try $R$ allele, have been shown to be impaired in their ability to survive inside cytokine-activated mouse and human macrophages $(12,19)$. Significantly, when the HB3 clone was electroporated with the plasmid pTTcTR, this recombinant strain (HB3-pTTcTR) regained its ability to resist
SNAP cytotoxicity to approximately wildtype levels. It is important to note that the HB3 mutant clone possesses only one tryR allele, whilst the parental clone (LV9-3) possesses three allelic copies of the gene (12). Despite this, the parental and all mutant lines used in the present study proliferated at equivalent rates under the conditions used.

The fact that the try $R$ mutant HB3 showed only a partial decrease in the survival rate ( $40 \%)$ when incubated with low doses of SNAP suggests that the remaining TryR activity present in this clone may still provide important antioxidant protection. This finding is consistent with the observation that Leishmania cells that overexpress a transdominant mutant version of try $R$ and as a result retain only about $15 \%$ of TryR activity, are not significantly impaired in their ability to metabolise hydrogen peroxide in vitro and are able to maintain wild-type levels of thiols under normal conditions of growth (34). It is possible that GSH may also contribute significantly to the residual antioxidant activity observed in clone HB3, as discussed above. In this context, we observed that all tryR mutant clones, despite having a reduction in TryR activities, present similar glutathione levels compared to $L$. donovani wild-type cells. This finding is in accordance with previous data demonstrating that $L$. donovani wild-type and try $R$ mutants with low levels of TryR catalytic activity did not differ in their levels of $\mathrm{T}(\mathrm{SH})_{2}$, glutathionyl-spermidine or glutathione (19, 35). Levels of reduced thiols (e.g., GSH, $\mathrm{T}(\mathrm{SH})_{2}$ ) may only become limiting under conditions of oxidative stress in the context of a compromised $\mathrm{T}(\mathrm{SH})_{2} /$ TryR antioxidant system $(12,19)$. An intriguing aspect of the $L$. donovani mutants that needs further investigation is that, in contrast to wild-type $L$. donovani (Figure 3), their sensitivity to SNAP was not dose-dependent.

Trypanosomatids also produce significant amounts of ovothiol A (36) which has recently been shown to catalytically acceler- 
ate nitrosothiol, both GSNO and $\mathrm{T}(\mathrm{SNO})_{2}$, decomposition to NO (37). The rate-limiting step in this reaction is the reduction of oxidised ovothiol which in Leishmania is mediated indirectly via trypanothione/trypanothione reductase, as is also the case for oxidized glutathione (GSSG) (5). Thus, we cannot exclude the possibility that the difference in cytotoxic potency of SNAP observed here between different tryR clones of Leishmania is dependent on variable ovothiol A concentrations and hence on nitrogen-free radical production.

The present data suggest that both antioxidant systems, glutathione and $\mathrm{T}(\mathrm{SH})_{2} /$
TryR, are involved in protecting Leishmania against the toxic effect of nitrogen-derived reactive species.

\section{Acknowledgments}

We thank Ms. Giuliana Bertozi, Ms. Ana Kátia dos Santos, and Ms. Fabíola Leslie Mestriner for technical assistance (FMRPUSP) and Jamil Assreuy (Department of Pharmacology, UFSC) for helping with nonprotein low-molecular weight thiol measurement. We are grateful to Professor A.H. Fairlamb (University of Dundee, UK) for providing $L$. donovani TryR mutants.

\section{References}

1. Molyneux DH (1997). Current public health status of the Trypanosomiases and Leishmaniases. In: Hide G, Mottran JC, Coombs GH et al. (Editors), Trypanosomiasis and Leishmaniasis: Biology and Control. CAB International, Oxford, UK.

2. Fairlamb AH, Blackburn $P$, Ulrich $P$ et al. (1985). Trypanothione: a novel bis (glutathionyl) permidine cofactor for glutathione reductase in trypanosomatids. Science, 227: 1485-1487.

3. Fairlamb AH \& Cerami A (1992). Metabolism and functions of trypanothione in the kinetoplastida. Annual Review of Microbiology, 46: 695-729.

4. Nogoceke E, Gommel DU, Kiess M et al. (1997). A unique cascade of oxidoreductases catalyses trypanothione-mediated peroxide metabolism in Crithidia fasciculata. Biological Chemistry, 378: 827-836.

5. Ariyanayagam MR \& Fairlamb AH (2001). Ovothiol and trypanothione as antioxidants in trypanosomatids. Molecular and Biochemical Parasitology, 115: 189-198.

6. Mukhopadhyay R, Dey S, Xu N et al. (1996). Trypanothione overproduction and resistance to antimonials and arsenicals in Leishmania tarentolae. Proceedings of the National Academy of Sciences, USA, 93: 10383-10387.

7. Liew FY, Li Y \& Millott $S$ (1990). Tumor necrosis factor- $\alpha$ synergizes with IFN- $\gamma$ in mediating killing of Leishmania major through the induction of nitric oxide. Journal of Immunology, 145: 4306-4310.

8. Liew FY, Millott S, Parkinson C et al. (1990). Macrophage killing of Leishmania parasite in vivo is mediated by nitric oxide from Larginine. Journal of Immunology, 144: 4794-4797.

9. Stenger $N$, Donhauser $H$, Thüring $M$ et al. (1996). Reactivation of latent leishmaniasis by inhibition of inducible nitric oxide synthase. Journal of Experimental Medicine, 183: 1501-1514.

10. Assreuy J, Cunha FQ, Epperlein M et al. (1994). Production of nitric oxide and superoxide by activated macrophages and killing of Leishmania major. European Journal of Immunology, 24: 672-676.

11. Romão PRT, Fonseca SG, Hothersall JS et al. (1999). Glutathione protects macrophages and Leishmania major against nitric oxidemediated cytotoxicity. Parasitology, 118: 559-566.
12. Tovar J, Wilkinson S, Mottram JC et al. (1998). Evidence that trypanothione reductase is an essential enzyme in Leishmania by targeted replacement of the tryA locus. Molecular Microbiology, 29: 653-660.

13. Tovar J \& Fairlamb AH (1986). Extrachromosomal, homologous expression of trypanothione reductase and its complementary mRNA in Trypanosoma cruzi. Nucleic Acids Research, 24: 29422949.

14. Kapler GM, Coburn CM \& Beverley SM (1990). Stable transfection of the human parasite Leishmania major delineates a 30-kilobase region sufficient for extrachromosomal replication and expression. Molecular and Cellular Biology, 10: 1084-1094.

15. Ellman GL \& Lysko H (1967). Disulphide and sulfhydryl compounds in TCA extracts of human blood and plasma. Journal of Laboratory and Clinical Medicine, 70: 518-527.

16. Tietze $F$ (1969). Enzymatic method for quantitative determination of nanogram amounts of total and oxidised glutathione: applications to mammalian blood and other tissues. Analytical Biochemistry, 27: 502-522.

17. Hothersall JS, Cunha FQ, Neild GH et al. (1997). Induction of nitric oxide synthesis in $\mathrm{J} 774$ cells lowers intracellular glutathione: effect of modulated glutathione redox status on nitric oxide synthase induction. Biochemical Journal, 322: 477-481.

18. Zhang $Y$ \& Hogg $N$ (2004). The mechanism of transmembrane $S-$ nitrosothiol transport. Proceedings of the National Academy of Sciences, USA, 101: 7891-7896.

19. Dumas C, Ouellette M, Tovar J et al. (1997). Disruption of the trypanothione reductase gene of Leishmania decreases its ability to survive oxidative stress in macrophages. EMBO Journal, 16: 25902598.

20. Lemesre JL, Sereno D, Daulouède $S$ et al. (1997). Leishmania spp: nitric oxide metabolic inhibition of promastigote and axenically grown amastigote forms. Experimental Parasitology, 86: 58-68.

21. Granger DL, Taintor R, Cook J et al. (1980). Injury of neoplastic cells by murine macrophages leads to inhibition of mitochondrial respira- 
tion. Journal of Clinical Investigation, 65: 357-370.

22. Hibbs Jr JB, Vavrin $Z$ \& Taintor RR (1987). L-arginine is required for expression of the activated macrophage effector mechanism causing selective metabolic inhibition in target cells. Journal of Immunology, 138: 550-565.

23. Minhas HS \& Thornalley PJ (1995). Reduced glutathione esters antidotes to toxicity. Cytotoxicity induced by hydrogen peroxide, 1chloro-2-4-dinitrobenzene, and menadione in murine $\mathrm{P}_{388 \mathrm{D}_{1}}$ macrophages in vitro. Journal of Biochemical Toxicology, 10: 245-250.

24. Augusto O, Bonini MG \& Trindade DF (2004). Spin trapping of glutathiyl and protein radicals produced from nitric oxide-derived oxidants. Free Radical Biology and Medicine, 36: 1224-1232.

25. Grinberg L, Fibach E, Amer J et al. (2005). N-acetylcysteine amide, a novel cell-permeating thiol, restores cellular glutathione and protects human red blood cells from oxidative stress. Free Radical Biology and Medicine, 38: 136-145.

26. Arrick BA, Griffith OW \& Cerami A (1981). Inhibition of glutathione synthesis as a chemotherapeutic strategy for trypanosomiasis. Journal of Experimental Medicine, 153: 720-725.

27. Yawetz A \& Agosin M (1980). Glutathione S-transferase and drug metabolism in Trypanosoma cruzi: in vivo and in vitro formation of thioethers. Comparative Biochemistry and Physiology. C, Comparative Pharmacology and Toxicology, 66C: 265-267.

28. Hurst JK \& Lymar SV (1995). Rapid reaction between peroxynitrite and carbon dioxide: implications for biological activity. Journal of the American Chemical Society, 117: 8867-8868.

29. Bonini MG, Radi R, Ferrer-Sueta et al. (1999). Direct EPR detection of the carbonate radical anion produced from peroxynitrite and carbon dioxide. Journal of Biological Chemistry, 274: 10802-10806.

30. Linares E, Giorgio S, Mortara RA et al. (2001). Role of peroxynitrite in macrophage microbicidal mechanisms in vivo revealed by protein nitration and hydroxylation. Free Radical Biology and Medicine, 30: 1234-1242.

31. Bonini MG \& Augusto $O$ (2001). Carbon dioxide stimulates the production of thyil, sulfinyl and disulphide radical anion from thiol oxidation by peroxynitrite. Journal of Biological Chemistry, 276: 9749-9754.

32. Radi R (2004). Nitric oxide, oxidants, and protein tyrosine nitration. Proceedings of the National Academy of Sciences, USA, 101: 40034008.

33. Lopes de Menezes S \& Augusto O (2001). EPR detection of glutathionyl and protein-tyrosyl radicals during the interaction of peroxynitrite with macrophages (J774). Journal of Biological Chemistry, 276: 39879-39884.

34. Tovar J, Cunningham ML, Smith AC et al. (1998). Down-regulation of Leishmania donovani trypanothione reductase by heterologous expression of a trans-dominant mutant homologue: Effect on parasite intracellular survival. Proceedings of the National Academy of Sciences, USA, 95: 5311-5316.

35. Kelly JM, Taylor MC, Smith K et al. (1993). Phenotype of recombinant Leishmania donovani and Trypanosoma cruzi which overexpress trypanothione reductase: sensitivity towards agents that are thought to induce oxidative stress. European Journal of Biochemistry, 218: 29-37.

36. Steenkamp DJ \& Spies HS (1994). Identification of a major lowmolecular-mass thiol of the trypanosomatid Crithidia fasciculata as ovothiol A. Facile isolation and structural analysis of the bimane derivative. European Journal of Biochemistry, 223: 43-50.

37. Vogt RN \& Steenkamp DJ (2003). The metabolism of S-nitrosothiols in the trypanosomatids: the role of ovothiol A and trypanothione. Biochemical Journal, 371: 49-59. 